\title{
The contribution of open extremity fractures to infection in multiply injured patients
}

\author{
W. Naziri' ${ }^{1}$, W. G. Cheadle ${ }^{1}$, D.:H. Livingston ${ }^{2}$, J. L. Rodriguez ${ }^{3}$, K. M. Starko ${ }^{4}$ and \\ H. C. Polk Jr ${ }^{1}$ \\ ${ }^{1}$ Department of Surgery, University of Louisville School of Medicine, Louisville, KY, USA, ${ }^{2}$ Trauma Service, UMDNJ - \\ University Hospital, Newark, NJ, USA, ${ }^{3}$ Trauma Burn Center, University of Michigan, Ann Arbor, MI, USA, and \\ ${ }^{4}$ Genentech Inc., South San Francisco, CA, USA
}

We sought to determine whether a contaminated open fracture was a reliable component for calculating the Outcome Predictive Score in patients with multiple injuries. We studied 41 patients whose primary source of contamination was open extremity fractures. Only one of the 41 patients developed osteomyelitis. The rate of infection from an open fracture is minimal in the multiply injured patient. Inclusion of patients with open fractures in studies that assess the likelihood of infection and the value of anti-infective agents incorrectly identified patients for clinical trials and results in an overestimation of survival based on the Outcome Predictive Score. These findings suggest that open fractures should be excluded as an entry criterion in future clinical trials.

\section{Introduction}

Since injury is a leading cause of morbidity and mortality in the USA, various injury assessment grading systems were developed to classify multiply injured patients prognostically into clinically relevant subgroups. All but one of the trauma assessment methods outlined in Table I (Committee

Presented as a poster at the Southeastern Surgical Congress, Tarpon Springs, Florida, 8-10 February 1993. on Medical Aspects of Automotive Safety, 1971; Baker et al., 1974; Knaus et al., 1985; Boyd et al., 1987; Hershman et al., 1988; Champion et al., 1989) emphasize physiological parameters. Although the severity of the acute physiological insult determines survivability in the immediate peritrauma period, secondary consequences of the physiological insult (e.g. infection) determine long-term morbidity and mortality.

Within the past decade, the knowledge of cellular immune functions has expanded, and it is clear that a profound immune suppression is associated with trauma and infection. Faist et al. (1986, 1987) described decreased immune response by circulating mononuclear cells in burned as well as multiply injured patients. Our laboratory (Cheadle et al., 1989) reported that human leukocyte antigen-DR (HLA-DR), a class II major histocompatibility antigen, was depressed in response to multiple injuries and/or infection. The expression of HLA-DR was determined to be an essential component in the ability of an antigen-presenting cell to present adequately an antigen to effector immune cells.

With this increased awareness of immune suppression in injured patients, the Outcome Predictive Score (OPS)

Table I. Various injury assessment grading systems

\begin{tabular}{|c|c|c|c|}
\hline Year & Author & Grading system & Description \\
\hline 1971 & $\begin{array}{l}\text { Committee on } \\
\text { Medical Aspects of } \\
\text { Automotive Safety }\end{array}$ & Abbreviated Injury Scale & $\begin{array}{l}\text { Developed to grade the injury severity of automotive injury victims through the } \\
\text { assignment of specific scores to each injury. In the past two decades, it has } \\
\text { evolved to include penetrating injuries. }\end{array}$ \\
\hline 1974 & Baker et al. & Injury Severity Score & $\begin{array}{l}\text { Derived from the Abbreviated Injury Scale to give a single value as the index of } \\
\text { severity of injury. }\end{array}$ \\
\hline 1985 & Knause et al. & APACHE II & $\begin{array}{l}\text { Represents an inclusive clinical assessment of intensive care patients without } \\
\text { regard to the nature or site of injury. }\end{array}$ \\
\hline 1987 & Boyd et al. & TRISS method & $\begin{array}{l}\text { The original Trauma Score, Revised Trauma Score, and Injury Severity Score } \\
\text { combined in order to increase specificity and sensitivity in predicting clinical } \\
\text { outcome in multiply injured patients. }\end{array}$ \\
\hline 1988 & Hershman et al. & Outcome Predictive Score & $\begin{array}{l}\text { Takes into account multiple factors, including age, the degree of contamination at } \\
\text { the time of injury, Injury Severity Score, and the extent of immune suppression as } \\
\text { a consequence of trauma as measured by HLA-DR expression on circulating } \\
\text { monocytes. }\end{array}$ \\
\hline 1989 & Champion et al. & Revised Trauma Score & $\begin{array}{l}\text { Based on physiological parameters for rapid assessment of injured individuals for } \\
\text { triage and outcome prediction. }\end{array}$ \\
\hline
\end{tabular}


(Hershman et al., 1988) was developed to incorporate HLA-DR expression as a measure of the immune system status into a system stratification of patients likely to develop infection and/or death. A common denominator in survivors of multiple injuries is infection, and the late mortality due to septic complications probably results from both contamination at the time of injury as well as host immune suppression.

The basic study was designed to evaluate the efficacy of interferon-gamma (IFN- $\gamma$ ) for the prevention of injuryassociated infections in a prospectively randomized population (Polk et al., 1992). One primary inclusion criterion for these patients was the existence of a contaminated wound, predisposing such patients to infection. Open extremity fractures (grade II or III) were defined as contaminated, thereby qualifying a substantial percentage of patients for inclusion in this study. Local infectious complications resulting from open fracture include acute infection of the open wound, requiring an adjustment in clinical management of the patient or late sequelae in the form of osteomyelitis.

Here, we present data that indicate that the rate of infection from an open fracture is a small contributor to sepsis in multiple injuries, and it contributes to an overestimation of the OPS for the purpose of assigning risk factors in these patients.

\section{Patients and methods}

A randomized, prospective, double-blind clinical trial was undertaken at four university medical centres (University of Louisville School of Medicine, University of Medicine and Dentistry in New Jersey, State University of New York at Buffalo, and University of Michigan) to determine the efficacy of IFN- $\gamma$ therapy for the prevention of injury-associated infection. A total of 213 patients were entered into the study, of which 212 were evaluated (Polk et al., 1992). Entry criteria for the study were: age over 15 years, an Injury Severity Score greater than 20, and bacterial contamination at one or more of the injury sites. If present, bacterial contamination was graded as $1+$ or $2+$; open extremity fractures (grade II or III) were graded as $1+$ (Table II). A second-generation cephalosporin was used initially in most patients but was modified as clinically justified for the appropriate treatment of specific bacterial infections. Antibiotic usage in those patients with open extremity fractures was left to the discretion of the orthopaedic surgeon in charge. Usually, penicillin G, cefazolin, and aminoglycoside were administered intravenously for at least 5 days.

Patients were randomized to treatment with IFN- $\gamma$ or placebo for 10 days and were followed for at least 90 days. Forty-one patients whose sole source of contamination was open extremity fractures (18 placebo, 23 IFN- $\gamma$ patients), were admitted to the study.

\section{Results}

Of the 41 patients with open extremity fractures, only one actually developed infection at the site of the open fracture, resulting in osteomyelitis. Osteomyelitis was defined by the need for operative excision of contaminated tissue and was based on an unequivocal clinical diagnosis that required a change in the pretreatment pattern.

Based on this information, we recalculated the OPS for day 3 of hospitalization after excluding open extremity
Table II. Contamination grading system

Examples of degree of bacterial contamination

\section{$2+$ Contamination}

Opening of colon and rectum

Degloving injury of the abdominal wall associated with enteric contamination.

Degloving injury of perineum or buttocks.

$1+$ contamination in addition to requirement for abdominal or pelvic packs or closure with prosthesis.

\section{$1+$ Contamination}

Opening of oesophagus, stomach, small bowel.

Degloving injury greater than 10 per cent total body surface area. Abdominal or pelvic packing.

Abdominal closure with prosthesis.

Clinical significant inhalation injury per bronchoscopy or requiring respiratory support.

Major pulmonary laceration.

Facial fractures into sinuses.

Grade II or III open extremity fractures.

Reprinted with permission from Polk H. C. Jr, Cheadle W. G., Livingston D. H. et al. (1992) A randomized prospective clinical trial to determine the efficacy of interferon-gamma in severely injured patients. Am. J. Surg. 163, 191.

Table III. Outcome Predictive Score on day 3 after injury

\begin{tabular}{|c|c|c|c|c|}
\hline \multirow[b]{2}{*}{ Treatment } & \multicolumn{2}{|c|}{$\begin{array}{l}\text { Open fractures } \\
\text { included }\end{array}$} & \multicolumn{2}{|c|}{$\begin{array}{l}\text { Open fractures } \\
\text { excluded }\end{array}$} \\
\hline & No. pts & OPS & No.pts & OPS \\
\hline \multicolumn{5}{|l|}{ Placebo } \\
\hline Uninfected Survivors & 59 & $160 \pm 84$ & 49 & 163 上 89 \\
\hline Infected Survivors & 27 & $234 \pm 87$ & 22 & $246 \pm 104$ \\
\hline Dead & 10 & $315 \pm 190$ & 9 & $324 \pm 199$ \\
\hline \multicolumn{5}{|l|}{ Interferon-gamma } \\
\hline Uninfected Survivors & 61 & $127 \pm 77$ & 50 & $125 \pm 77$ \\
\hline Infected Survivors & 31 & $166 \pm 97$ & 24 & $173 \pm 95$ \\
\hline Dead & 9 & $233 \pm 107$ & 6 & $209 \pm 112$ \\
\hline
\end{tabular}

No. pts = number of patients; OPS = Outcome Predictive Score.

fracture patients (Table III). Three days after admission, 197 of the total study population had an HLA-DR assay performed, including 37 of the 41 patients with open extremity fractures. There was a uniform increase in the OPS in the placebo group when the open fracture patients were excluded from the criteria, but no increase was seen in the IFN- $\gamma$ group.

\section{Discussion}

With better medical technology and care of injured patients, an ever-increasing percentage of these patients survive the initial resuscitation effort and continue to require intensive care for prolonged periods of time. Infection is the most common complication in survivors of multiple injury.

Trauma assessment scores, by virtue of their design, are intended to identify survivors and nonsurvivors of acute injury. The OPS is a useful development to identify survivors of multiple injuries who are at subsequent risk of major infection and its complications. Such a scoring system is not helpful for clinical decision-making but is also 
important for identifying those individuals who are suitable for further studies of anti-infective therapy and those who ought to be excluded from such studies.

Infectious complications are the most common sequelae of multiple injuries, and the development of such infections often can be traced to contamination at the site of the wound at the time of injury. Haemorrhage, shock, and the extent of contamination of an open extremity wound are important prerequisites in the development of infection. In order to carry out clinical trials of anti-infective agents, there needs to be a systematic way to stratify patients into groups that can be reliably identified as high or low risk for the development of infection. The OPS was developed to address this specific issue in survivors of polytrauma. The OPS takes into account multiple factors including the degree of contamination at the time of injury and the extent of immune suppression as a consequence of trauma as measured by HLD-DR expression on circulating monocytes. Forty-one multiply injured patients met inclusion criteria for contamination by virtue of grade II or III open extremity fractures, but only one developed significant acute or delayed infection at the site of the open fracture. In contrast, others have previously found that initial contamination at the fracture sitc is an important predictor of subsequent local infection (Merritt, 1988; Roth et al., 1986). More aggressive and repetitive surgical irrigation and wound cleaning may have contributed to the low incidence of osteomyelitis seen in this trial. Whether this low rate of infection of open fractures is due to the intrinsic efficacy of broad-spectrum antibiotics employed here is not known (Braun et al., 1987; Seligson and Henry, 1991).

The role of IFN- $\gamma$ in the development of osteomyelitis of local infection at the site of an open fracture is difficult to assess in the light of only one case of infection. Further studies have been performed at other medical centres evaluating IFN- $\gamma$ in multiple injuries, and their results are pending. After excluding open extremity fracture patients in recalculating the OPS on day 3 of hospitalization, there was a noticeable uniform increase in OPS in all placebo groups. The results were not consistent in patients treated with IFN- $\gamma$ because IFN- $\gamma$ alters HLA-DR expression and therefore alters the OPS. This uniform increase in OPS, though not statistically significant, was evident in all three placebo-treated groups, indicating that the inclusion of open extremity fractures as a criterion of contamination led to overestimation of the OPS.

As financial resources become more scarce in carrying out clinical trials, and as clinical trials become more costly, it will be extremely important to identify correctly patients appropriate for clinical trials evaluating the efficacy of anti-infective agents. While inclusion of contaminated wounds, especially those in the abdominal cavity, are important in predicting the development of septic complications, the inclusion of open extremity fractures (grade II or III) as contaminated wounds may lead to inclusion of patients with minimal likelihood of development of infectious complications from such wounds. For this reason, open extremity fractures as the sole source of contamination should be excluded as a criterion of contamination in predicting future infections in clinical trials of anti-infective agents.

\section{References}

Baker S. P., O'Neil B., Haddon W., Jr et al. (1974) The injury severity score: a method for describing patients with multiple injuries and evaluating emergency care. J. Trauma 14, 187.

Boyd C. R., Tolson M. A. and Copes W. S. (1987) Evaluating traumatic care: the TRISS method. J. Trauma 27, 370.

Braun R., Enzler M. A. and Rittman W.W. (1987) A double-blind clinical trial of prophylactic cloxacillin in open fractures. J. Orthop. Trauma 1, 12.

Champion H. R., Sacco W. J., Copes W. S. et al. (1989) A revision of the trauma score. J. Trauma 29,623.

Cheadle W. G., Wilson M., Hershman M. J. et al. (1989) Comparison of trauma assessment scores and their use in prediction of infection and death. Ann. Surg, 209, 541.

Committee on Medical Aspects of Automotive Safety (1971) Rating the severity of tissue damage. I. The abbreviated scale. J.A.M.A. 215, 277.

Faist E., Mewes A., Strasser T. et al. (1987) Alteration of monocyte function following major injury. Arch. Surg. 123, 287.

Faist E., Kupper T. S., Baker C. L. et al. (1986) Depression of cellular immunity after major injury. Arch. Surg. 121, 1000.

Hershman M. J., Cheadle W. G., Kuftinec D. et al. (1988) An outcome predictive score for sepsis and death following injury. Injury 19, 263.

Knaus W. A., Draper E. A., Wagner D. P. et al. (1985) APACHE II: a severity of disease classification system. Crit. Care Med. 13, 818 .

Merritt K. (1988) Factors increasing the risk of infection in patients with open fractures. J. Trauma 28, 823 .

Polk H. C. Jr, Cheadle W. G., Livingston D. H. et al. (1992) A randomized prospective clinical trial to determine efficacy of IFN-gamma in severely injured patients. Am. J. Surg. 163, 191.

Roth I., Fry D. E. and Polk H. C. Jr (1986) Infectious morbidity in extremity fractures. J. Trauma 26, 757.

Seligson D. and Henry S. L. (1991) Treatment of compound fractures. Am. J. Surg. 161, 693.

Paper accepted I December 1993.

Requests for reprints should be addressed to: Hiram C. Polk, Jr, MD, Department of Surgery, University of Louisville, Louisville, Kentucky 40292, USA. 